\title{
Impact of irradiated methane ice crusts on compositional interpretations of TNOs (Research Note)
}

\author{
R. Brunetto ${ }^{1,2, \star}$ and T. L. Roush ${ }^{3}$ \\ 1 Dipartimento di Fisica, Università del Salento, via Arnesano, 73100 Lecce, Italy \\ 2 INAF - Osservatorio Astrofisico di Catania, via S. Sofia 78, 95123 Catania, Italy \\ e-mail: rosario.brunetto@ias.u-psud.fr \\ 3 NASA Ames Research Center, MS 245-3, Moffett Field, CA 94035-1000, USA
}

Received 21 October 2007 / Accepted 19 February 2008

\begin{abstract}
Context. Minor bodies in the outer solar system show a wide variety of spectral colors related to both composition and surface processing, e.g. cosmic ion irradiation.

Aims. We investigate the effect of an irradiation mantle on the reflectance spectrum in the V-NIR (visible-near-infrared) range. In particular we investigate the condition needed for a weathering crust to mask the presence of water ice.

Methods. We start from laboratory experiments of ion irradiated methane ice to study the optical properties of the hydrocarbon residue by-product of the space weathering process. We compare the real and imaginary index of refraction with those of Titan and ice tholins and with those of amorphous carbon. We use the estimated optical constants to model a layered configuration using the Hapke theory, varying the thickness and grain size of the modeled crust.

Results. We find that a relatively thin (tens of microns) crust of irradiated methane by-products can mask the presence of water ice bands in the spectrum, while a much larger layer of tholins would be required to produce a similar effect. We also estimate the conditions for the detection of water ice on trans-Neptunian objects (TNOs). We discuss the results in view of the astrophysical timescale.
\end{abstract}

Key words. astrochemistry - Kuiper Belt - Oort Cloud - solar system: general - methods: laboratory

\section{Introduction}

Outer solar system bodies show a great variety of spectral colors (Doressoundiram et al. 2002). This can be explained by both compositional and space weathering (SW) effects (Moroz et al. 2004; Brunetto et al. 2006), in competition with rejuvenating processes, such as impacts or internal activity (Stern 2002; Cook et al. 2007).

In particular, cosmic ion irradiation on Centaurs and transNeptunian objects (TNOs) is believed to induce both chemical and structural modification of ices, with formation of different species and by-products (Palumbo et al. 1999; Moore et al. 2003; Brunetto et al. 2005), and amorphization of crystalline water (Mastrapa \& Brown 2006). When carbon is present, an hydrocarbon residue is formed that may produce a crust of refractory material, as in the case of comets in the Oort cloud (Strazzulla et al. 1991).

The SW processes mentioned above induce color variations that can reproduce the observed spectral variety of Centaurs and TNOs (Brunetto et al. 2006). The hydrocarbon refractories, in particular, are generally characterized by low albedo and red spectral slope. These components are thought to be present on the majority of outer solar system bodies, with the noteworthy exception of $2003 \mathrm{EL}_{61}$ and its associated carbon-depleted population (Brown et al. 2007). If the object develops an irradiation

\footnotetext{
* Present address: Institut d'Astrophysique Spatiale, UMR-8617, Université Paris-Sud, bâtiment 121, 91405 Orsay Cedex, France.
}

mantle, it is possible that this will spectrally mask the presence of pristine molecules underneath, like water or methane.

Here we investigate the effect of the production of an irradiation mantle on the reflectance spectrum in the V-NIR range, using both experimental and modeling results. In particular we investigate the condition for a SW crust to mask the presence of underlying water ice.

\section{Experiments and models}

In the interpretation of spectra of TNOs and Centaurs, an important role is played by experimental studies able to simulate the SW processes and characterize the corresponding spectral variations.

We start from laboratory experiments of ion irradiation of methane ice to study the optical properties of the hydrocarbon residue by-product. The experimental apparatus used is at the Laboratory of Experimental Astrophysics at the INAFOsservatorio Astrofisico di Catania (Italy). In a stainless steel vacuum chamber $\left(<10^{-7}\right.$ mbar $)$ a $\mathrm{KBr}$ substrate is placed in thermal contact with the cold finger of a closed-cycle helium cryostat $(10-300 \mathrm{~K})$. The substrate is surrounded by a cold trap within which the pressure is lower by at least an order of magnitude (less than $10^{-8}$ mbar). Gaseous methane was prepared in a prechamber and admitted by a gas inlet, through a needle valve, into the vacuum chamber, where it deposited onto a $\mathrm{KBr}$ substrate. 


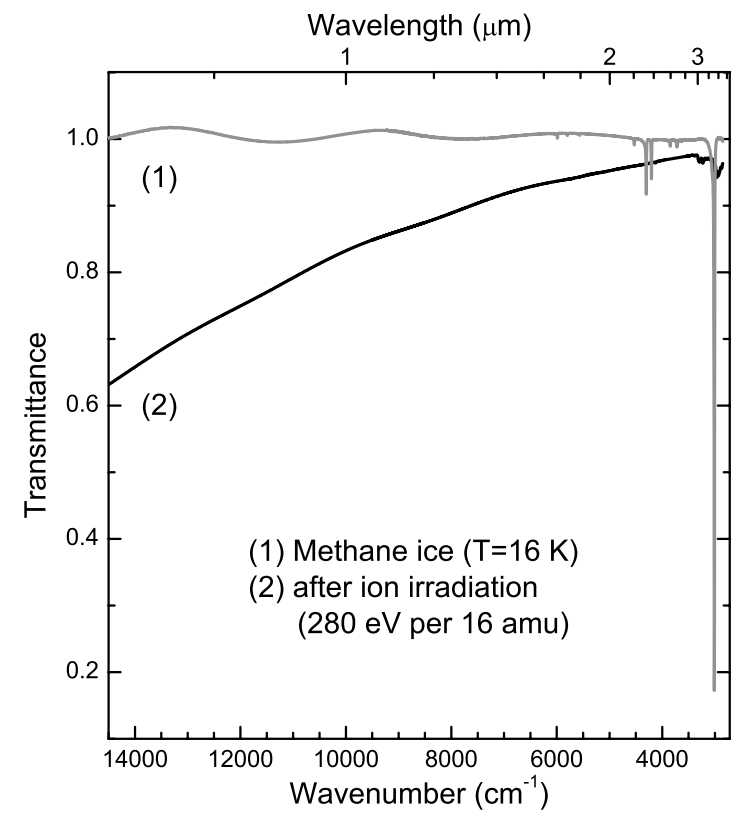

Fig. 1. Transmittance spectra of methane ice, deposited at $16 \mathrm{~K}$, before and after irradiation (dose of $280 \pm 50 \mathrm{eV}$ per $16 \mathrm{amu}$ ).

A FTIR spectrophotometer (Bruker Equinox 55) views the $\mathrm{KBr}$ substrate, through $\mathrm{KBr}$ windows, such that the sample forms angles of $45^{\circ}$ both with the V-NIR light and with the ion beam. As a result it is possible to collect spectra and irradiate the sample without rotating the cold finger. This experimental arrangement allows collection of in situ transmission spectra in two distinct and partially overlapping ranges $(\approx 14500-8550$ and $\left.10000-2800 \mathrm{~cm}^{-1}\right)$, altogether covering the $0.69-3.57 \mu \mathrm{m}$ spectral range. A rotatable polarizer is placed after transmission through the sample and it was used to collect spectra in the P polarization, i.e. with the electric vector parallel to the plane of incidence. Thus to model the observations it was necessary to separate polarization states (this was important to estimate the optical constants, see below). The spectral resolution was $4 \mathrm{~cm}^{-1}$ in the NIR and $8 \mathrm{~cm}^{-1}$ in the visible. Simultaneously, the sample can be irradiated with $30-400 \mathrm{keV}$ ions. Other details can be found in Brunetto et al. (2006).

A layer of methane ice $(1.16 \pm 0.1 \mu \mathrm{m}$ thick $)$ was deposited at $T=16 \mathrm{~K}$, and then irradiated with $\mathrm{H}^{+}$and $\mathrm{Ar}^{+}$ions (energy of $200 \mathrm{keV}$ ) to a dose of $280 \pm 50 \mathrm{eV}$ per $16 \mathrm{amu}$, as estimated using the SRIM code at http://wWw.SRIM.org/ (Ziegler et al. 1985). The icy sample was monitored using transmittance spectroscopy, before and after irradiation. Spectra are shown in Fig. 1.

The thickness of the originally deposited ice was measured using the interference pattern of the spectral continuum, from the knowledge of the refractive index of pristine methane (Pearl et al. 1991; Martonchik \& Orton 1994; Grundy et al. 2002). The value obtained was verified to be correct within $10 \%$, by comparing it with a separate thickness estimate obtained from using the areas of the methane absorption bands.

After ion irradiation, a hydrocarbon residue is formed, whose thickness is different from the pristine layer. Using the SRIM code, we estimated that the amount of sputtered material is less than $0.5 \mathrm{~nm}$, i.e. negligible. The main cause of variation in the thickness is the increase in the density, due to the formation of a different structure. The density of solid methane at low temperatures is $\approx 0.46 \mathrm{~g} / \mathrm{cm}^{3}$. Calcagno et al. (1985) measured a density of $\approx 0.5 \mathrm{~g} / \mathrm{cm}^{3}$ for a residue obtained after ion irradiation

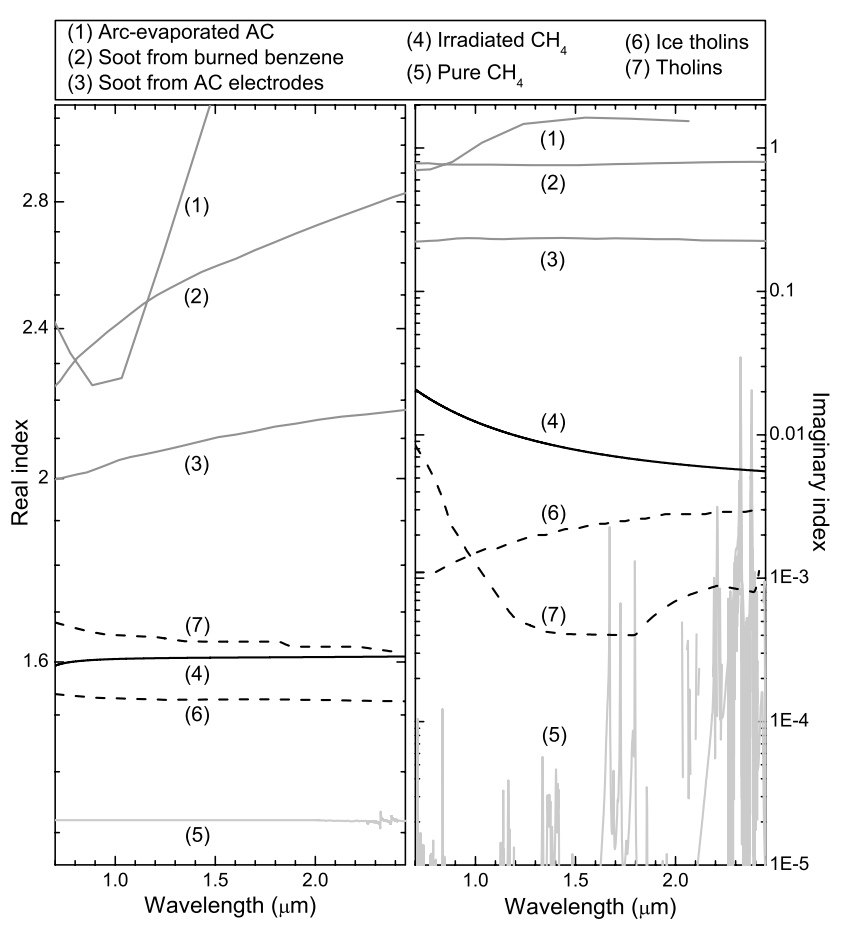

Fig. 2. The real (left panel) and imaginary (right panel) parts of the refractive index, for arc-evaporated carbon film (Palik 1991), soot from burned benzene (Rouleau \& Martin 1991), soot from AC electrodes (Rouleau \& Martin 1991), $\mathrm{ICH}_{4}$ (this work), pure $\mathrm{CH}_{4}$ (Pearl et al. 1991; Grundy et al. 2002), ice tholins (Khare et al. 1993), and Titan tholins (Khare et al. 1984). Uncertainties are: ice tholin, $\pm 20 \%$ where $k>10^{-2}, \pm 50 \%$ where $k<10^{-2}, \pm 0.01$ for $n$; Titan tholin, $\pm 30 \%$ for $k, \pm 3 \%$ for $n$; arc-evaporated carbon film, $k$ values highly reliable for $k>0.02$, no statement about $n$. Rouleau \& Martin (1991) provide no estimates of the uncertainty.

of solid $\mathrm{CH}_{4}$. Here we assume a density $0.5 \mathrm{~g} / \mathrm{cm}^{3}$ of the irradiated material. The sample thickness is reduced proportionally $(\approx 10 \%)$ to $\approx 1.05 \pm 0.1 \mu \mathrm{m}$.

The optical constants, real $(n)$ and imaginary $(k)$ indices of refraction, of irradiated methane $\left(\mathrm{ICH}_{4}\right)$ were estimated from the transmittance spectra (at $T=16 \mathrm{~K}$ ) joining the two different and partially overlapping ranges. A computer procedure incorporates a Kramers-Kronig analysis, following the presentation of Hudgins et al. (1993), and the equations of transmission and reflection, using a matrix approach for the film and the substrate (Heavens 1970) that easily allows distinguishing between $\mathrm{P}$ and $\mathrm{S}$ polarization. Iterative steps are performed until the $n$ and $k$ converge towards stable values. Uncertainties in the film thickness lead to errors in the estimated optical constants of about $5 \%$ in $n$ and $20 \%$ in $k$.

We stress that $\mathrm{ICH}_{4}$ is not a simple ice. In fact, there is little, if any, ice left after irradiation, and the remaining residue is a complex hydrocarbon. The optical properties of carbonaceous refractory materials strongly depend on the initial composition, synthesis conditions, and energy involved (Imanaka et al. 2004; Moroz et al. 2004). In Fig. 2 we compare the optical constants of $\mathrm{ICH}_{4}$ derived here to those of carbonaceous compounds created by various energetic processes, in particular Titan (Khare et al. 1984) and ice tholins (Khare et al. 1993), that are two materials commonly used in the models of minor bodies spectra (Cruikshank et al. 2005), and different types of amorphous carbon. 
We note that $\mathrm{ICH}_{4}$ (Fig. 2, curve 4, at the dose we used) has real and imaginary indices that lie in between those of tholins (curves 6 and 7) and amorphous carbon (curves 1-3). Ferini et al. (2004) monitored irradiation of frozen methane using Raman spectroscopy; they found that at low dose (up to about $380 \mathrm{eV}$ per $16 \mathrm{amu}$ ), the sample shows an intense fluorescence signal due to the formation of a damaged molecular solid; at high dose (up to about $820 \mathrm{eV}$ per $16 \mathrm{amu}$ ) hydrogen is progressively lost and the Raman feature of amorphous carbon appears. Thus, the dose we use here (about $280 \mathrm{eV}$ per $16 \mathrm{amu}$ ) indicates that $\mathrm{ICH}_{4}$ has not yet evolved towards a black carbon, as confirmed by Fig. 2 .

We caution the reader about the relatively large uncertainties in our estimated values of $n$ and $k$. A more accurate derivation of the optical constants should consider a direct measure of the thickness of the residue inside the vacuum chamber. However, this is a difficult task owing to the fact that the sample after irradiation is much more absorbing than pristine methane. Nonetheless, valuable information can be obtained regarding the influence of such a refractory material by using the values derived here in an astrophysical context.

\section{Results}

Given the significant vapor pressure of methane, even at the low temperatures in the outer solar system, it is easy to envision the physical geometry where a layer of methane occurs on a substrate of less volatile material. This layered configuration could result regardless of whether the methane is endogenous or exogenous to a solid body.

In order to mimic the SW of TNOs, we use the optical constants estimated above to model a layered configuration using the Hapke theory. The model calculates the reflectance from a system where a layer overlies an infinitely thick substrate. The input parameters are the optical constants of the upper layer and substrate materials. We use the equations $7.45 \mathrm{c}, 8.89,9.14$, and 11.24 of Hapke (1993).

Relevant parameters that can be changed are the thickness (up- $t$ ) and effective grain size (up- $d_{\mathrm{e}}$ ) of the upper layer, and the effective grain size $\left(10-d_{\mathrm{e}}\right)$ of the substrate. The model also allows varying the filling factor $(f)$ of the upper layer, which is simulating a non-uniformly distributed crust, e.g. patchy.

We first investigate the conditions for the SW crust to entirely mask the presence of underlying water ice. We use the optical constants of crystalline water ice from Warren $(1984)(T \approx$ $266 \mathrm{~K}$ ) at wavelengths $<1 \mu \mathrm{m}$, because no data are available at lower temperatures at the shorter wavelengths investigated here, and from Grundy \& Schmitt (1998) $(T=40 \mathrm{~K})$ at the longer wavelengths. Results are shown in Fig. 3, where we also compare a crust of $\mathrm{ICH}_{4}$ with a crust of tholins. Water ice is characterized by the presence of two bands at about 1.5 and $2.0 \mu \mathrm{m}$; in addition, crystalline water ice at low temperature shows a narrower feature at about $1.65 \mu \mathrm{m}$.

We find that a $10 \mu \mathrm{m}$ layer of $\mathrm{ICH}_{4}$ is already enough to mask the presence of water ice bands in the spectrum. In the case of tholins, a larger thickness (about $100 \mu \mathrm{m}$ ) is required to produce a similar effect; this is explained by the fact that in the NIR the imaginary part of the refractive index of $\mathrm{ICH}_{4}$ is about 10 times larger than that of Titan tholins (see Fig. 2). Note that the $\mathrm{ICH}_{4}$ models also produce a very low albedo, which is consistent with observations of many outer solar system bodies characterized by dark surfaces.

To illustrate the implications of the model for compositional interpretation of telescopic data, we fix two detection limits for

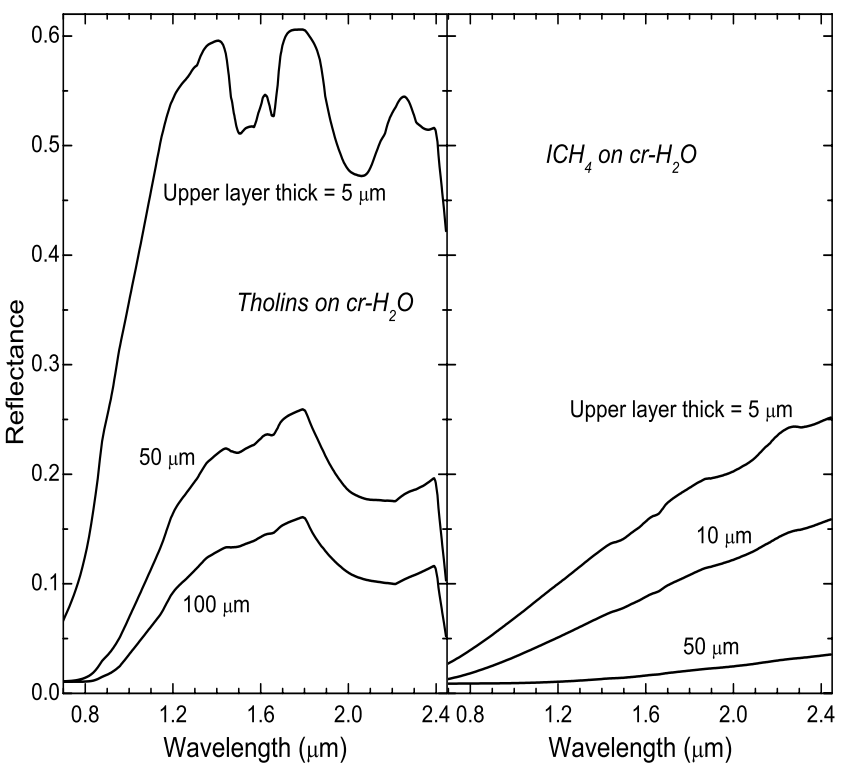

Fig. 3. Synthetic reflectance spectra of a two-layer system, with crystalline water ice on the bottom $(T=40 \mathrm{~K}$, grain size of $20 \mu \mathrm{m})$. Right and left panels are for an overlying layer of $\mathrm{ICH}_{4}$ and Titan tholins, respectively. Different upper layer thickness $t$ are indicated for each curve (we fix up- $d_{\mathrm{e}}=t$ ) and the filling factor $f$ is fixed at $100 \%$ for all curves.

Table 1. Detection conditions for water ice bands. Upper layer: $\mathrm{ICH}_{4}$ at $16 \mathrm{~K}$. Lower layer: crystalline $\mathrm{H}_{2} \mathrm{O}$ at $40 \mathrm{~K}$.

\begin{tabular}{cccccc}
\hline \hline$f$ & up- $t$ & up- $d_{\mathrm{e}}$ & lo- $d_{\mathrm{e}}$ & $3 \%$ det. & $10 \%$ det. \\
\hline $80 \%$ & $5 \mu \mathrm{m}$ & $5 \mu \mathrm{m}$ & $20-200 \mu \mathrm{m}$ & yes & yes \\
$100 \%$ & $?^{*}$ & $?^{*}$ & $20-200 \mu \mathrm{m}$ & yes & yes \\
$100 \%$ & $5 \mu \mathrm{m}$ & $5 \mu \mathrm{m}$ & $20-200 \mu \mathrm{m}$ & yes & no \\
$100 \%$ & $10 \mu \mathrm{m}$ & $10 \mu \mathrm{m}$ & $20-200 \mu \mathrm{m}$ & no & no \\
\hline
\end{tabular}

$* d_{\mathrm{e}}<5 \mu \mathrm{m}$ would violate the Hapke theory in this range.

Table 2. Detection conditions for water ice bands. Upper layer: Titan tholins. Lower layer: crystalline $\mathrm{H}_{2} \mathrm{O}$ at $40 \mathrm{~K}$.

\begin{tabular}{cccccc}
\hline \hline$f$ & up- $t$ & up- $d_{\mathrm{e}}$ & lo- $d_{\mathrm{e}}$ & $3 \%$ det. & $10 \%$ det. \\
\hline $80 \%$ & $50 \mu \mathrm{m}$ & $50 \mu \mathrm{m}$ & $20-200 \mu \mathrm{m}$ & yes & yes \\
$100 \%$ & $20 \mu \mathrm{m}$ & $20 \mu \mathrm{m}$ & $20-200 \mu \mathrm{m}$ & yes & yes \\
$100 \%$ & $50 \mu \mathrm{m}$ & $50 \mu \mathrm{m}$ & $20-200 \mu \mathrm{m}$ & yes & no \\
$100 \%$ & $100 \mu \mathrm{m}$ & $100 \mu \mathrm{m}$ & $20-200 \mu \mathrm{m}$ & no & no \\
\hline
\end{tabular}

the substrate water ice bands; when the band depth is $3 \%$ or $10 \%$ deep with respect to the reflectance of the continuum (e.g. Clark \& Roush 1984). We then calculate the spectra for differing values of; 1) grain sizes for the crust and substrate particles; 2) thickness of the crust; and 3) the filling factor of the material forming the crust.

Results of our analysis are reported in Tables 1 and 2, showing detection conditions at $3 \%$ and $10 \%$ error bars for water ice bands, when water ice is covered by a crust of $\mathrm{ICH}_{4}$ (Table 1) or Titan tholins (Table 2). Again, we find that tholins require a thickness about 10 times larger than $\mathrm{ICH}_{4}$ to mask the water ice bands. Note that a $5 \mu \mathrm{m}$ crust of $\mathrm{ICH}_{4}$ would allow water ice to be detected with a $3 \%$ sensitivity, but not at $10 \%$ sensitivity. In this case, if one wants to detect water ice bands with a $10 \%$ sensitivity, the crust should probably be thinner than $5 \mu \mathrm{m}$. However, up- $d_{\mathrm{e}}=5 \mu \mathrm{m}$ is for a crust grain diameter of $5 \mu \mathrm{m}$ and a thinner crust would represent a physically impossible situation, i.e. crust cannot be thinner than the grains composing it. Additionally, to 
set up- $d_{\mathrm{e}}<5 \mu \mathrm{m}$ approaches the particle size regime that begins to violate the underlying geometric optics assumptions of Hapke theory at the longest wavelength in the spectral range shown. Even though the geometric optics assumption may be valid at the shorter wavelengths where the water ice features are more prominent, the calculated continuum levels used to determine the band depth at the longer wavelength are affected.

\section{Discussion}

As discussed by Strazzulla et al. (2003), multiple ion sources contribute to the total energetic ion environment in the outer solar system and include solar energetic particles (SEP), anomalous cosmic rays (ACR), and galactic cosmic rays (GRC). Strazzulla et al. (2003) discuss how the fluxes from these sources, and their associated energy distributions, are different at various locations in the solar system. SEP have a roughly inverse square relationship with heliocentric distance while GCR become more important at greater heliocentric distances. To estimate the timescale of crust formation we use Fig. 6 from Strazzulla et al. (2003). We find that a $5 \mu \mathrm{m}$ surface layer would accumulate $280 \mathrm{eV}$ per $16 \mathrm{amu}$ (the dose used in our experiment) in a timescale of about $3 \times 10^{10}$ years at $40 \mathrm{AU}$; however this decreases to about $3 \times 10^{9}$ years at $85 \mathrm{AU}$. If the object spends time in the very local interstellar medium, then the timescale decreases to about $6 \times 10^{7}$ years. These are very rough estimates, with errors of the order of a factor of 10, due to the large number of uncertainties affecting the calculation. Solar ultraviolet radiation provides additional energy for alteration of the original ices (Cruikshank et al. 1998), although it is relatively weak, requires long timescales, and chiefly influences the near surface, i.e. $\approx 100 \mathrm{~nm}$ for Ly- $\alpha$ photons and using the $k$ values for $\mathrm{CH}_{4}$ of Martonchik \& Orton (1994).

Such long timescales associated with the formation of a $5 \mu \mathrm{m}$ crust may prove difficult for preservation of this crust against micrometeoritic impacts, especially for classical Kuiper Belt Objects (KBO). However, we note the two general population of impactors, interstellar and KBO dust grains, are likely to contain their own component of pristine and altered carbonaceous materials that can be delivered to the surfaces (Cruikshank et al. 1998). Those materials surviving the impact events will be incorporated into the upper $\mathrm{cm}$ to meters of the surface and re-exposed during subsequent impacting events perhaps forming a layer on the underlying substrate, as is consistent with the layered model proposed here.

In any case, it is clear that the orbital elements and their evolution are critical parameters to the formation of a crust. Determining ion, UV, and micrometeorite fluxes as a function of time for specific TNOs is beyond the scope of the current study, but we can estimate that the process of crust formation and water masking are more efficient for comets in the Oort Cloud than classical KBOs.

\section{Conclusions}

We investigated some effects of SW by energetic ions on TNOs. In particular we studied the optical properties of the residue obtained after irradiating methane ice in the laboratory. We compared the real and imaginary index of refraction with those of Titan and ice tholins, and those of amorphous carbon.
The calculated optical constants were used to model a layered configuration using Hapke theory, varying the thickness and grain size of the modeled crust. This allowed us to investigate the effects of the production of an irradiation mantle on the reflectance spectrum in the V-NIR range. In particular we studied the condition required for a SW crust to mask the presence of underlying water ice.

We found that a relatively thin (tens of microns) crust of irradiated methane ice can mask the presence of water ice bands in the spectrum, while a much thicker layer of tholins would be required to produce a similar effect. Although the exact details will vary, the model results are broadly applicable to substrate materials other than water ice.

In future studies, efforts should concentrate on the estimation of optical constants of refractory material synthesized from icy mixtures relevant to the chemistry of TNOs, e.g. $\mathrm{CH}_{4}, \mathrm{H}_{2} \mathrm{O}, \mathrm{N}_{2}$, $\mathrm{CH}_{3} \mathrm{OH}$.

Acknowledgements. The authors are grateful to G. A. Baratta, M. E. Palumbo, G. Strazzulla, and V. Orofino for their precious help and suggestions. T.L.R. acknowledges support from NASA's Planetary Geology and Geophysics program.

\section{References}

Brown, M. E., Barkume, K. M., Ragozzine, D., \& Schaller, E. L. 2007, Nature, 446, 294

Brunetto, R., Baratta, G. A., Domingo, M., \& Strazzulla, G. 2005, Icarus, 175, 226

Brunetto, R., Barucci, M. A., Dotto, E., \& Strazzulla, G. 2006, ApJ, 644, 646 Calcagno, L., Foti, G., \& Strazzulla, G. 1985, Nuovo Cimento D Serie, 5, 255 Clark, R. N., \& Roush, T. L. 1984, J. Geophys. Res., 89, 6329

Cook, J. C., Desch, S. J., Roush, T. L., Trujillo, C. A., \& Geballe, T. R. 2007, ApJ, 663, 1406

Cruikshank, D. P., Roush, T. L., Bartholomew, M. J., et al. 1998, Icarus, 135, 389 Cruikshank, D. P., Imanaka, H., \& Dalle Ore, C. M. 2005, Adv. Space Res., 36, 178

Doressoundiram, A., Peixinho, N., de Bergh, C., et al. 2002, AJ, 124, 2279

Ferini, G., Baratta, G. A., \& Palumbo, M. E. 2004, A\&A, 414, 757

Grundy, W. M., \& Schmitt, B. 1998, J. Geophys. Res., 103, 25809

Grundy, W. M., Schmitt, B., \& Quirico, E. 2002, Icarus, 155, 486

Hapke, B. 1993, Theory of Reflectance and Emittance Spectroscopy (New York: Cambridge Univ. Press)

Heavens, O. 1970, Thin Film Physics (London: Methuen \& Co., Ltd.)

Hudgins, D. M., Sandford, S. A., Allamandola, L. J., \& Tielens, A. G. G. M. 1993, ApJS, 86, 713

Imanaka, H., Khare, B. N., Elsila, J. E., et al. 2004, Icarus, 168, 344

Khare, B. N., Sagan, C., Arakawa, E. T., et al. 1984, Icarus, 60, 127

Khare, B. N., Thompson, W. R., Cheng, L., et al. 1993, Icarus, 103, 290

Martonchik, J. V., \& Orton, G. S. 1994, Appl. Opt., 33, 8306

Mastrapa, R. M. E., \& Brown, R. H. 2006, Icarus, 183, 207

Moore, M. H., Hudson, R. L., \& Ferrante, R. F. 2003, Earth Moon and Planets, 92, 291

Moroz, L., Baratta, G., Strazzulla, G., et al. 2004, Icarus, 170, 214

Palik, E. D. 1991, Handbook of optical constants of solids II (Boston: Academic Press)

Palumbo, M. E., Castorina, A. C., \& Strazzulla, G. 1999, A\&A, 342, 551

Pearl, J., Ngoh, M., Ospina, M., \& Khanna, R. 1991, J. Geophys. Res., 96, 17476

Rouleau, F., \& Martin, P. G. 1991, ApJ, 377, 526

Stern, S. A. 2002, AJ, 124, 2297

Strazzulla, G., Baratta, G. A., Johnson, R. E., \& Donn, B. 1991, Icarus, 91, 101 Strazzulla, G., Cooper, J. F., Christian, E. R., \& Johnson, R. E. 2003, C. R. Phys., 4,791

Warren, S. G. 1984, Appl. Opt., 23, 1206

Ziegler, J. F., Biersack, J. P., \& Littmark, U. 1985, The Stopping and Range of Ions in Solids (New York: Pergamon Press) 